\title{
Vagal Modification can be a Valid Predictor of Late Recurrence of Paroxysmal Atrial Fibrillation Independent of the Pulmonary Vein Isolation Technique
}

\author{
Takumi Yamada, MD; Naoki Yoshida, MD*; Yoshimasa Murakami, MD**; \\ Taro Okada, MD**; Yukihiko Yoshida, MD ${ }^{\dagger}$; Masahiro Muto, MD*; \\ Yasuya Inden, MD*; Toyoaki Murohara, MD*
}

\begin{abstract}
Background: Vagal denervation during pulmonary vein isolation (PVI) may reduce paroxysmal atrial fibrillation (PAF) recurrences. Valid predictors of late PAF recurrence after PVI were examined.

Methods and Results: Successful segmental (S-PVI) or circumferential PVI (C-PVI) was achieved in 120 consecutive PAF patients. The 24-h Holter recordings were obtained before and after PVI. Logistic regression analysis was performed to determine independent predictors of PAF recurrence among the patient characteristics, PVI technique and heart rate variability (HRV). Of the 120 patients, 19 were excluded from analysis because of additional ablation for recurrent PAF after successful PVI. Among the remaining 101 patients, 68 were free from PAF (Group-I) and 33 had late PAF recurrences (Group-II) at 1-year follow-up. The incidence of PAF recurrence and conduction recovery in isolated PVs revealed in the second session was significantly lower for C-PVI (21.6\% and $28.1 \%)$ than for S-PVI $(44.0 \%$ and $58.3 \%)(\mathrm{P}<0.05)$. There were no significant differences in the HRV highfrequency component (HF) reflecting parasympathetic activity or the low/high frequency component (LF/HF) reflecting sympathetic activity between the 2 techniques. By multivariate analysis, the PVI technique, HF and $\mathrm{LF} / \mathrm{HF}$ were independent predictors of $\mathrm{PAF}$ recurrence $(\mathrm{P}<0.05)$.
\end{abstract}

Conclusions: Vagal denervation may prevent late PAF recurrence independent of the PVI technique. (Circ J 2009; 73: $1606-1611)$

Key Words: Atrial fibrillation; Autonomic nervous system; Heart rate variability; Pulmonary vein isolation; Radiofrequency catheter ablation

$\mathbf{P}$ ulmonary vein isolation (PVI) is an effective technique for curing atrial fibrillation (AF),,-8 and it has been reported that adding an ablation to the PV ablation that is aimed at vagal denervation adequate for eliminating vagal reflexes ${ }^{9}$ or targeting the ganglionated plexi in the left atrium (LA) ${ }^{10}$ may reduce $\mathrm{AF}$ recurrence. Those reports suggest that modification of the autonomic nervous function may prevent AF recurrence after PVI. PVI alone has been proved to induce an immediate decrease in autonomic nervous function, ${ }^{11,12}$ but the relationship between modification of the autonomic nervous function and AF recurrence after PVI remains unclear, because multiple factors may be associated with AF recurrence. This study was undertaken to investigate whether or not vagal denervation is an independent predictor of $\mathrm{AF}$ recurrence after PVI.

\section{Methods}

\section{Study Population}

The study population consisted of 120 consecutive patients
(93 men, 59 \pm 11 years; range 29-80 years) with symptomatic paroxysmal AF (PAF) refractory to $3 \pm 1$ class I or III antiarrhythmic drugs (not including amiodarone). The mean PAF history was $4 \pm 4$ years $(0-17)$. The mean echocardiographic dimension of the LA was $35 \pm 6 \mathrm{~mm}(25-46)$ and mean left ventricular ejection fraction was $67 \pm 9 \%$ (50-89). The exclusion criteria were sick sinus syndrome, diabetes mellitus, thyroid dysfunction, recent myocardial infarction ( $<6$ months), history of a prior thoracotomy, $\beta$-blocker therapy, and a pacing rhythm. The first 60 patients underwent segmental PVI (S-PVI) and the second 60 patients underwent circumferential PVI (C-PVI). These patients were retrospectively examined. Each patient gave written informed consent, and all antiarrhythmic drugs were discontinued for at least 5 half-lives prior to the study.

\section{Electrophysiologic Study}

A 7-French decapolar catheter with 1-5-1-mm interelectrode spacing between each electrode pair (St Jude Medical, AF Division, Minnetonka, MN, USA) was deployed into the coronary sinus via the subclavian vein. The transseptal

(Received March 10, 2009; accepted April 27, 2009; released online July 17, 2009)

Division of Cardiovascular Disease, University of Alabama at Birmingham, Birmingham, AL, USA, *Department of Cardiology, Nagoya University Graduate School of Medicine, Nagoya, **Division of Cardiology, Aichi Prefectural Cardiovascular and Respiratory Center, Ichinomiya and Division of Cardiology, Nagoya Dai-ni Red Cross Hospital, Cardiovascular Center, Nagoya, Japan

Mailing address: Takumi Yamada, MD, Division of Cardiovascular Disease, University of Alabama at Birmingham, VH B147, 1670 University Boulevard, 1530 3rd AVE S, Birmingham, AL 35294-0019, USA. E-mail: takumi-y@ fb4.so-net.ne.jp

All rights are reserved to the Japanese Circulation Society. For permissions, please e-mail: cj@j-circ.or.jp 
procedure was performed with intracardiac echocardiography guidance recorded with a 9-French transducer catheter (Boston Scientific, Natick, MA, USA) operating at $9 \mathrm{MHz}$. Catheterization into the LA was performed with a 1-puncture and 2-sheath technique (one sheath (8-French, St Jude Medical) for an ablation catheter and another (8.5-French, Soft Tip EP Sheath ${ }^{\mathrm{TM}}$, EP Technologies, Boston Scientific Corporation, San Jose, CA, USA) for a mapping catheter). After the transseptal procedure, systemic anticoagulation was achieved with intravenous heparin to maintain an activated clotting time $>300$ s. Selective angiography of the PVs was performed in all patients.

\section{PV Mapping}

The left superior PV, left inferior PV, right superior PV and right inferior PV were all targeted for 2 PVI techniques. A 31-mm multielectrode basket catheter (MBC) (Constellation $^{\mathrm{TM}}$, EP Technologies), which consisted of 8 splines $(\mathrm{A}-\mathrm{H})$ with 8 1-mm electrodes at 2-mm spacing, was deployed within 3 to 4 PVs via the atrial septum. An MBC was introduced toward the distal PV and then pulled back as proximally as possible without dislodgement under fluoroscopic guidance until its most proximal electrodes were positioned at the PV ostium or antrum, which was identified by selective angiogram. A total of 56 bipolar electrograms were recorded by the MBC during sinus rhythm (right PVs) or distal coronary sinus pacing (left PVs). When AF persisted during the electrophysiologic study, internal cardioversion was used to restore sinus rhythm and an $\mathrm{MBC}$ recording of at least 1 beat was obtained during the appropriate rhythm. If an MBC could not be deployed in the inferior PVs, a 20-electrode circular catheter (Lasso ${ }^{\mathrm{TM}}$, Biosense Webster, Diamond Bar, CA, USA) was used for mapping those PVs.

\section{Catheter Ablation}

S-PVI targeting a preferential electrical connection between the PVs and LA was performed as previously described. ${ }^{4}$ For 3-dimensional PV mapping using an MBC, the onset of a longitudinal activation pattern toward the distal PVs with the earliest activation at the circumference of the LA-PV junction was identified as a prior electrical connection. Radiofrequency (RF) energy was delivered with a target temperature of $55^{\circ} \mathrm{C}$ and maximum power output of $40 \mathrm{~W}$ for $60 \mathrm{~s}$ (EPT-1000TC generator ${ }^{\mathrm{TM}}$, EP Technologies), using an 8-mm tip catheter (Blazer II 5770T ${ }^{\mathrm{TM}}$, EP Technologies). C-PVI targeting the PV antrum potentials was performed as previously described. ${ }^{6} \mathrm{RF}$ energy was delivered in the same manner as in the S-PVI. If a residual conduction gap was detected after PV antrum ablation, additional RF applications to the PV side just next to the previous RF lesions were delivered. The final end-point of the 2 PVI techniques was defined as either the abolition or dissociation of the distal PV potentials independent of the elimination of vagal reflexes. After successful PVI, induction of spontaneous AF was attempted with burst atrial pacing and an isoproterenol infusion. When spontaneous AF remained inducible, isolation of the superior vena cava (SVC) for SVC triggers, or focal ablation targeting the other triggers, was performed as previously reported. ${ }^{13,14}$ Those procedures above were repeated until the non-inducibility of spontaneous AF was confirmed.

\section{Analysis of the Heart Rate Variability (HRV)}

Serial 24-h Holter recordings were obtained at baseline, immediately and 1-, 3-, 6-, 12-months after the PVI to analyze the HRV. After an automatic analysis, the data file was visually reviewed and edited by an experienced technician. The heart rate and time- and frequency-domains HRV were analyzed from the Holter recordings using an analysis program (Philips Zymed Holter 2010 Plus). Supraventricular premature beats, AF, ventricular premature beats, electrical noise, and other aberrant ECG signals were excluded from the HRV analysis. The underlying rhythm was carefully analyzed, and only artifact-free episodes of sinus rhythm were included in further analyses. The HRV was used as an indicator of autonomic activity in accordance with the guidelines for standardization. ${ }^{15}$ The time-domain measures of the HRV included the standard deviation (SD) of all NN intervals (SDNN), SD of the averages of the NN intervals in all 5-min segments (SDANN), mean of the SD of all NN intervals for all 5-min segments (ASDNN), and root mean square successive differences (rMSSD). The frequency-domain measures of the HRV included the lowfrequency (LF: 0.04-0.15 Hz) power, high-frequency (HF: $0.15-0.40 \mathrm{~Hz})$ power, and ratio of the LF to HF powers (LF/HF). The frequency-domain HRV was calculated by a fast Fourier transform for each 5-min segment of data. All values of the frequency-domain HRV were expressed as the average of all 5-min segments of the 24-h recordings and were logarithmically transformed to avoid the undue influence of extreme values. The rMSSD and HF have been used to reflect parasympathetic nervous activity, and the LF/HF has been used to reflect sympathetic nervous activity.

\section{Follow-up}

The patients remained hospitalized under continuous rhythm monitoring for at least 3 days. During the follow-up period, no antiarrhythmic drugs were administered to any of the patients. Clinical follow-up was performed at 2 weeks, 1 month and every month until 12 months after the procedure, using 24-h Holter and cardiac recordings. All patients who reported symptoms were given an event monitor to document the cause of the symptoms. Multislice computed tomography was performed in all the patients 3 and 6 months after the procedure for the detection of PV stenosis.

\section{Statistical Analysis}

All values are expressed as the mean \pm SD. The frequencydomain measurements of the HRV (LF and HF) were expressed in squared milliseconds. Comparisons of continuous variables were analyzed using the Student's t-test. Categorical variables expressed as numbers and percentages in different groups were compared with a chi-square test. Multivariate logistic regression analysis was performed to determine the independent predictors of AF recurrence. Statistical significance was considered to be present at $\mathrm{P}<0.05$.

\section{Results}

The clinical characteristics of the patients undergoing S-PVI and C-PVI are shown in Table 1. There were no significant differences between the 2 groups in age, sex, duration of PAF, number of ineffective antiarrhythmic drugs, incidence of structural heart disease, echocardiographic LA dimension, or left ventricular ejection fraction.

\section{Catheter Ablation}

In all the study patients, successful isolation of all 4 PVs 
Table 1. Patient Characteristics

\begin{tabular}{lccc}
\hline & $\begin{array}{c}\text { S-PVI } \\
(\mathrm{n}=60)\end{array}$ & $\begin{array}{c}\text { C-PVI } \\
(\mathrm{n}=60)\end{array}$ & P value \\
\hline Age, years & $59 \pm 12$ & $60 \pm 10$ & $\mathrm{NS}$ \\
Sex, M/F & $46 / 14$ & $47 / 13$ & $\mathrm{NS}$ \\
Duration of PAF, years & $4 \pm 4$ & $5 \pm 4$ & $\mathrm{NS}$ \\
Ineffective AADs, $\mathrm{n}$ & $2 \pm 1$ & $3 \pm 1$ & $\mathrm{NS}$ \\
Structural heart disease, $\mathrm{n}$ & 2 & 3 & $\mathrm{NS}$ \\
LAD, mm & $34 \pm 5$ & $35 \pm 5$ & $\mathrm{NS}$ \\
LVEF, \% & $68 \pm 8$ & $67 \pm 8$ & $\mathrm{NS}$ \\
\hline
\end{tabular}

S-PVI, segmental pulmonary vein isolation (PVI); C-PVI, circumferential PVI; M/F, male/female; PAF, paroxysmal atrial fibrillation; AAD, antiarrhythmic drug; LAD, left atrial dimension; LVEF, left ventricular ejection fraction.

was achieved. During S-PVI and C-PVI, vagal reflexes such as transient AV block, sinus bradycardia, or hypotension were elicited in 5 and 7 patients, respectively. In 19 patients (S-PVI=10, C-PVI=9), spontaneous AF was induced after successful PVI and additional SVC isolation and/or focal ablation targeting the triggers was performed. All 19 cases were excluded from the analysis in case additional ablation affected autonomic nervous function. There were still no significant differences in the clinical characteristics of the remaining 50 patients with S-PVI compared with the 51 with C-PVI.

Among the 101 patients, 68 (28 with S-PVI, 40 with C-PVI; Group-I) were free from symptomatic PAF without any antiarrhythmic drugs and 33 (22 with S-PVI, 11 with C-PVI; Group-II) had late PAF recurrence at 1-year follow-
Table 2. Comparisons of HR and HRV Before Ablation

\begin{tabular}{lccc}
\hline & \multicolumn{2}{c}{ Late recurrence } & \\
\cline { 2 - 3 } & $\begin{array}{c}\text { No, } \\
\text { Group-I }\end{array}$ & $\begin{array}{c}\text { Yes, } \\
\text { Group-II }\end{array}$ & \\
\hline Minimum HR, beats/min & $47.1 \pm 7.5$ & $46.9 \pm 6.3$ & 0.54 \\
Average HR, beats/min & $67.1 \pm 9.2$ & $68.6 \pm 7.3$ & 0.66 \\
Maximum HR, beats/min & $118.8 \pm 17.7$ & $117.4 \pm 17.2$ & 0.75 \\
PACs, \% & $2.6 \pm 2.5$ & $2.8 \pm 2.5$ & 0.70 \\
ASDNN, ms & $63.2 \pm 16.8$ & $65.1 \pm 18.2$ & 0.95 \\
SDANN, ms & $119.2 \pm 29.0$ & $113.5 \pm 25.3$ & 0.57 \\
SDNN, ms & $149.3 \pm 38.6$ & $142.3 \pm 32.3$ & 0.68 \\
rMSSD, ms & $60.1 \pm 29.9$ & $62.9 \pm 39.2$ & 0.73 \\
ln LF, ms ${ }^{2}$ & $8.99 \pm 0.53$ & $8.86 \pm 0.45$ & 0.62 \\
ln HF, ms & $9.27 \pm 0.53$ & $9.14 \pm 0.54$ & 0.95 \\
LF/HF & $0.75 \pm 0.22$ & $0.77 \pm 0.17$ & 0.38 \\
\hline
\end{tabular}

$\mathrm{HR}$, heart rate; $\mathrm{HRV}$, heart rate variability; $\mathrm{PAC}$, premature atrial contraction; ASDNN, mean of the standard deviation of all NN intervals for all 5-min segments; SDANN, standard deviation of the averages of the NN intervals in all 5-min segments; SDNN, standard deviation of all NN intervals; rMSSD, root mean square successive differences; ln, natural logarithm; LF, low-frequency power; $\mathrm{HF}$, high-frequency power; $\mathrm{LF} / \mathrm{HF}$, the ratio of lowfrequency to high-frequency power.

up. In $10(45.4 \%)$ and $18(81.8 \%)$ of the 22 Group II patients with S-PVI, PAF recurred within 1 and 3 months after PVI, whereas PAF did not recur in any of the Group II patients with C-PVI within 3 months after PVI. The PAF recurrence rate was significantly higher in the patients with S-PVI than in those with C-PVI (44.0\% vs $21.6 \%, \mathrm{P}<0.05)$. The second procedure was performed in 23 (15 with S-PVI, 8 with C-PVI) of 33 Group-II patients. It was found that the
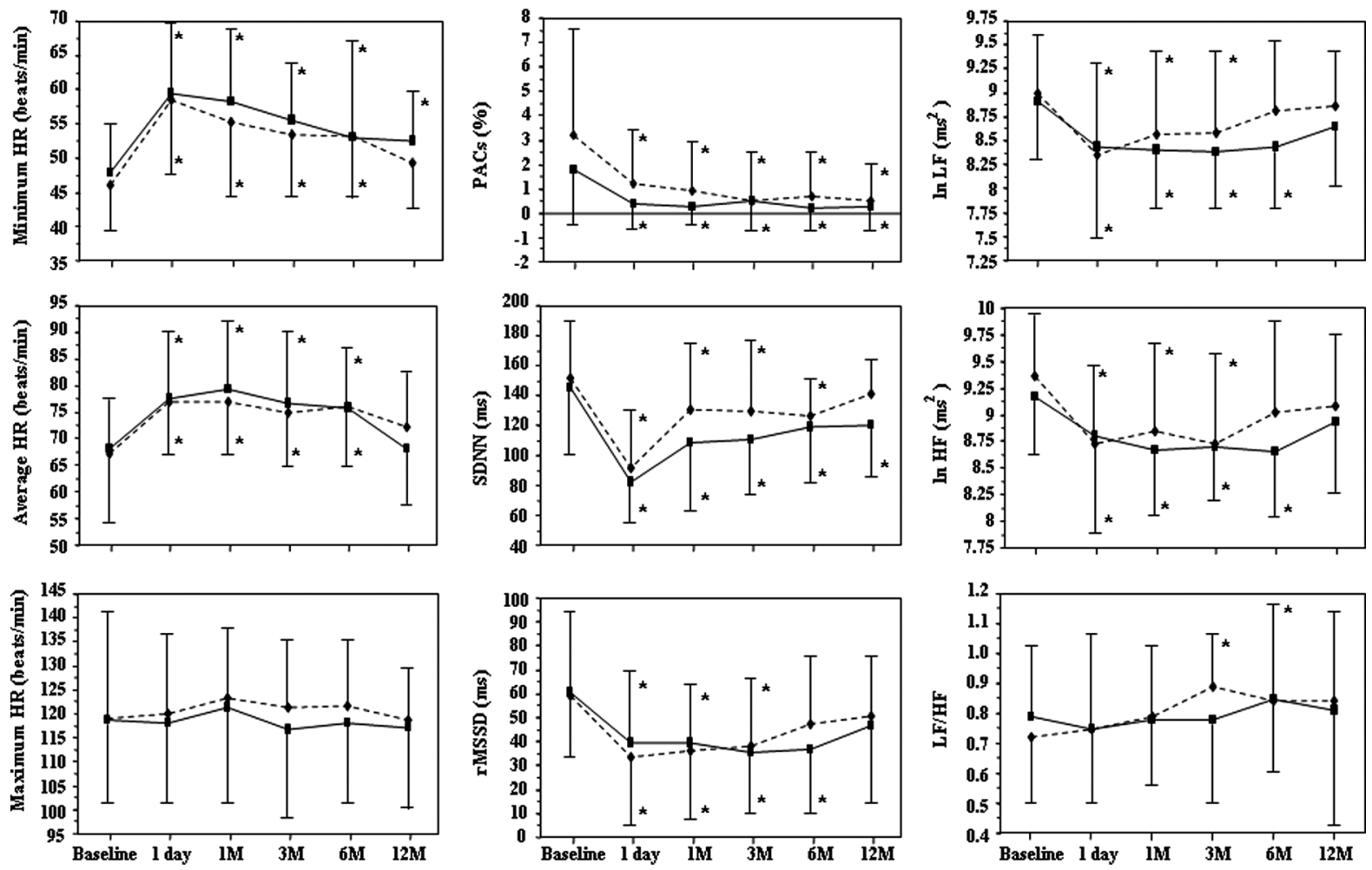

Figure. Serial changes in heart rate (HR), percentage of premature atrial contractions (PACs), and HR variability (HRV) before, immediately (1 day), 1 month $(1 \mathrm{M}), 3$ months $(3 \mathrm{M}), 6$ months $(6 \mathrm{M})$, and 12 months $(12 \mathrm{M})$ after pulmonary vein isolation (PVI) in the Group I patients. The solid lines indicate the circumferential PVI group data and dotted lines the segmental PVI group data. Other abbreviations as in Table 2 . $* \mathrm{P}<0.05$ vs before the ablation. 
Table 3. Comparison of the Changes in HR and HRV Before and After Ablation in Group-I

\begin{tabular}{|c|c|c|c|c|c|c|}
\hline & Before ablation & 1 day & 1 month & 3 months & 6 months & 12 months \\
\hline Minimum HR, beats/min & $47.1 \pm 7.5$ & $58.6 \pm 10.1 *$ & $58.3 \pm 9.9 *$ & $55.4 \pm 8.5 *$ & $53.0 \pm 12.9 *$ & $52.6 \pm 5.5^{*}$ \\
\hline Average HR, beats/min & $67.1 \pm 9.2$ & $76.8 \pm 10.4 *$ & $79.2 \pm 11.5^{*}$ & $76.5 \pm 13.6^{*}$ & $75.9 \pm 10.0^{*}$ & $68.2 \pm 9.1$ \\
\hline Maximum HR, beats/min & $118.8 \pm 17.7$ & $117.2 \pm 19.2$ & $121.2 \pm 18.6$ & $116.8 \pm 16.7$ & $117.9 \pm 14.2$ & $116.9 \pm 15.0$ \\
\hline PACs, $\%$ & $2.6 \pm 2.5$ & $0.8 \pm 1.6^{*}$ & $0.3 \pm 0.3^{*}$ & $0.5 \pm 1.0 *$ & $0.2 \pm 0.5^{*}$ & $0.3 \pm 0.7 *$ \\
\hline ASDNN, ms & $63.2 \pm 16.8$ & $34.4 \pm 19.2 *$ & $37.7 \pm 14.2^{*}$ & $40.1 \pm 15.6^{*}$ & $40.1 \pm 15.2^{*}$ & $42.2 \pm 13.2 *$ \\
\hline SDANN, ms & $119.2 \pm 29.0$ & $76.7 \pm 25.0 *$ & $100.8 \pm 40.4$ & $99.9 \pm 27.4$ & $109.8 \pm 33.3$ & $108.7 \pm 28.0$ \\
\hline SDNN, ms & $149.3 \pm 38.6$ & $91.4 \pm 33.8 *$ & $108.9 \pm 41.6^{*}$ & $110.9 \pm 29.2 *$ & $118.9 \pm 33.6^{*}$ & $120.0 \pm 28.6^{*}$ \\
\hline $\mathrm{rMSSD}, \mathrm{ms}$ & $60.1 \pm 29.9$ & $34.6 \pm 18.3^{*}$ & $39.2 \pm 21.0 *$ & $35.3 \pm 26.5^{*}$ & $36.9 \pm 27.0 *$ & $46.4 \pm 32.0$ \\
\hline $\ln \mathrm{LF}, \mathrm{ms}^{2}$ & $8.99 \pm 0.53$ & $8.37 \pm 0.86^{*}$ & $8.40 \pm 0.65^{*}$ & $8.39 \pm 0.48 *$ & $8.43 \pm 0.58 *$ & $8.65 \pm 0.55$ \\
\hline $\ln \mathrm{HF}, \mathrm{ms}^{2}$ & $9.27 \pm 0.53$ & $8.73 \pm 0.78 *$ & $8.67 \pm 0.59 *$ & $8.70 \pm 0.45^{*}$ & $8.66 \pm 0.62 *$ & $8.94 \pm 0.72$ \\
\hline $\mathrm{LF} / \mathrm{HF}$ & $0.75 \pm 0.22$ & $0.75 \pm 0.29$ & $0.78 \pm 0.19$ & $0.78 \pm 0.26$ & $0.85 \pm 0.31$ & $0.81 \pm 0.34$ \\
\hline
\end{tabular}

$* \mathrm{P}<0.05$ vs before ablation. Abbreviations as in Table 2 .

Table 4. Comparisons of HR and HRV in the Immediate Aftermath of Ablation

\begin{tabular}{lccc}
\hline & \multicolumn{2}{c}{ Late recurrence } & \\
\cline { 2 - 3 } & $\begin{array}{c}\text { No, } \\
\text { Group I } \\
(\mathrm{n}=68)\end{array}$ & $\begin{array}{c}\text { Yes, } \\
\text { Group II } \\
(\mathrm{n}=33)\end{array}$ & P value \\
& $58.6 \pm 10.1$ & $58.0 \pm 8.7$ & 0.74 \\
\hline Minimum HR, beats/min & $76.8 \pm 10.4$ & $80.0 \pm 10.0$ & 0.13 \\
Average HR, beats/min & $117.2 \pm 19.2$ & $118.8 \pm 14.9$ & 0.66 \\
Maximum HR, beats/min & $0.8 \pm 1.6$ & $1.9 \pm 2.5$ & 0.005 \\
PACs, \% & $34.4 \pm 19.2$ & $43.4 \pm 22.9$ & 0.03 \\
ASDNN, ms & $76.7 \pm 25.0$ & $81.0 \pm 29.1$ & 0.43 \\
SDANN, ms & $91.4 \pm 33.8$ & $105.3 \pm 42.1$ & 0.06 \\
SDNN, ms & $34.6 \pm 18.3$ & $59.1 \pm 28.6$ & $<0.0001$ \\
rMSSD, ms & $8.37 \pm 0.86$ & $8.65 \pm 0.94$ & 0.12 \\
ln LF, ms ${ }^{2}$ & $8.73 \pm 0.78$ & $9.24 \pm 0.95$ & 0.003 \\
ln HF, ms ${ }^{2}$ & $0.75 \pm 0.29$ & $0.62 \pm 0.23$ & 0.018 \\
LF/HF & & & \\
\hline Abrinnn & & & \\
\hline
\end{tabular}

Abbreviations as in Table 2.

incidence of electrical connection recovery between the LA and PV in the isolated PVs was significantly higher in the S-PVI patients $(58.3 \%)$ than in the C-PVI patients $(28.1 \%)$ $(\mathrm{P}<0.05)$ and there were no significant differences in the incidence of non-PV PAF foci between the S-PVI (33.3\%) and C-PVI (25.0\%) patients.

\section{Heart Rate and HRV Changes}

There were no significant differences in heart rate, percentage of premature atrial contractions (PACs) of the total beats on the Holter recordings (\% PACs) or HRV parameters before PVI among the patients undergoing S-PVI or C-PVI and with or without late PAF recurrence (Table 2). The results of the heart rate, \% PACs and HRV parameters in the Group-I patients are shown in Figure and Table 3. In those patients, the minimum and average heart rates increased immediately after either PVI technique and remained elevated for 6-12 months. The time- and frequency-domain HRV parameters, including the SDNN, rMSSD, and HF, decreased immediately after either PVI and remained attenuated for 3-12 months. The LF/HF did not change significantly immediately after either PVI, but increased late after S-PVI alone. The \%PACs had an acute reduction immediately after either PVI and then a subsequent gradual decrease throughout the entire observation period. The results of the heart rate, \%PACs and HRV parameters in the Group-II patients are shown in Table 4. In those patients, the minimum and average heart rates increased and the SDANN, SDNN and LF/HF decreased immediately after either PVI. The other time- and frequency-domain HRV
Table 5. Comparisons of HR and HRV at 3 Months After Ablation

\begin{tabular}{lccc}
\hline & \multicolumn{2}{c}{ Late recurrence } & \\
\cline { 2 - 3 } & $\begin{array}{c}\text { No, } \\
\text { Group I } \\
(\mathrm{n}=68)\end{array}$ & $\begin{array}{c}\text { Yes, } \\
\text { Group II } \\
(\mathrm{n}=10)\end{array}$ & \\
& P value \\
Minimum HR, beats/min & $55.4 \pm 8.5$ & $51.6 \pm 7.5$ & 0.13 \\
Average HR, beats/min & $76.5 \pm 13.6$ & $73.8 \pm 8.7$ & 0.35 \\
Maximum HR, beats/min & $116.8 \pm 16.7$ & $119.4 \pm 11.9$ & 0.41 \\
PACs, \% & $0.5 \pm 1.0$ & $0.8 \pm 1.3$ & 0.39 \\
ASDNN, ms & $40.1 \pm 15.6$ & $56.4 \pm 24.5$ & 0.01 \\
SDANN, ms & $99.9 \pm 27.4$ & $101.1 \pm 25.5$ & 0.77 \\
SDNN, ms & $110.9 \pm 29.2$ & $131.1 \pm 25.5$ & 0.04 \\
rMSSD, ms & $35.3 \pm 26.5$ & $62.3 \pm 40.9$ & 0.01 \\
ln LF, ms ${ }^{2}$ & $8.39 \pm 0.48$ & $8.83 \pm 0.52$ & 0.04 \\
ln HF, ms ${ }^{2}$ & $8.70 \pm 0.45$ & $9.19 \pm 0.75$ & 0.04 \\
LF/HF & $0.78 \pm 0.26$ & $0.74 \pm 0.28$ & 0.65 \\
\hline Abrynn & & & \\
\hline
\end{tabular}

Abbreviations as in Table 2.

parameters did not change significantly after either PVI. Because 27 of 33 (81.8\%) Group-II patients underwent another ablation procedure $(\mathrm{n}=23)$ and/or took antiarrhythmic drugs or $\beta$-blockers $(n=13)$ because of PAF recurrence, the subsequent Holter recordings after those interventions were excluded from the HRV analyses in those patients.

Comparisons of heart rate, \%PACs and HRV parameters in the 2 groups in the immediate aftermath of PVI are shown in Table 4. The rMSSD and HF, which reflect parasympathetic nervous activity, were significantly lower in Group-I than in Group-II (rMSSD: $34.6 \pm 18.3$ vs $59.1 \pm 28.6 \mathrm{~ms}$, P< 0.0001 ; ln HF: $8.73 \pm 0.78$ vs $\left.9.24 \pm 0.95 \mathrm{~ms}^{2}, \mathrm{P}=0.003\right)$. The LF/HF ratio, which reflects sympathetic nervous activity, was significantly higher in Group-I than in Group-II $(0.75 \pm 0.29$ vs $0.62 \pm 0.23, \mathrm{P}=0.018)$. The $\% \mathrm{PACs}$ was significantly lower in Group-I than in Group-II $(0.8 \pm 1.6$ vs $1.9 \pm 2.5 \%, \mathrm{P}=0.005)$. There was a significant correlation between $\mathrm{HF}$ and \%PACs in the immediate aftermath of PVI $(r=0.427, \mathrm{P}<0.0001)$. There were no significant differences between the 2 groups in the minimum, average, or maximum heart rate.

Comparisons of heart rate, \%PACs and HRV parameters in the 2 groups at 3 months after PVI are shown in Table 5. The rMSSD and HF were significantly lower in Group-I than in Group-II (rMSSD: $35.3 \pm 26.5$ vs $62.3 \pm 40.9 \mathrm{~ms}$, P< 0.01 ; ln HF: $8.703 \pm 0.45$ vs $\left.9.19 \pm 0.75 \mathrm{~ms}^{2}, \mathrm{P}=0.04\right)$. There were no significant differences between the 2 groups in the $\mathrm{LF} / \mathrm{HF}$ ratio, \% PACs, or minimum, average, or maximum heart rate. 
Table 6. Comparison of HR and HRV in the Immediate Aftermath of Either PVI Technique

\begin{tabular}{lccl}
\hline & $\begin{array}{c}\text { S-PVI } \\
(\mathrm{n}=50)\end{array}$ & $\begin{array}{c}\text { C-PVI } \\
(\mathrm{n}=51)\end{array}$ & P value \\
\hline Minimum HR, beats/min & $57.1 \pm 9.6$ & $59.6 \pm 9.7$ & 0.16 \\
Average HR, beats/min & $78.1 \pm 9.7$ & $77.4 \pm 11.0$ & 0.74 \\
Maximum HR, beats/min & $121.2 \pm 18.1$ & $114.4 \pm 17.4$ & 0.04 \\
PACs, \% & $1.8 \pm 2.5$ & $0.5 \pm 0.9$ & 0.0004 \\
ASDNN, ms & $43.5 \pm 23.1$ & $31.2 \pm 16.2$ & 0.001 \\
SDANN, ms & $83.5 \pm 29.3$ & $73.0 \pm 22.2$ & 0.03 \\
SDNN, ms & $108.5 \pm 43.1$ & $83.7 \pm 25.0$ & 0.0002 \\
rMSSD, ms & $47.6 \pm 29.2$ & $38.3 \pm 23.0$ & 0.06 \\
ln LF, ms ${ }^{2}$ & $8.51 \pm 1.00$ & $8.40 \pm 0.78$ & 0.53 \\
ln HF, ms & $8.96 \pm 1.04$ & $8.80 \pm 0.67$ & 0.30 \\
LF/HF & $0.70 \pm 0.27$ & $0.72 \pm 0.29$ & 0.63 \\
\hline
\end{tabular}

Abbreviations as in Table 2.

\section{Predictors of AF Recurrence}

Because there were no significant differences between the 2 PVI techniques in the ln HF or LF/HF ratio in the immediate aftermath of PVI (Table 6), these 2 parameters were enrolled in the multivariate analysis together with the PVI technique. By multivariate analysis, the PVI technique, high $\mathrm{HF}$, and low LF/HF ratio were independent predictors of late PAF recurrence $(\mathrm{P}<0.05$, all $)$ (Table 7). Multivariate analysis was not performed using the $\mathrm{HF}$ and $\mathrm{LF} / \mathrm{HF}$ ratio at 3 months after the PVI because the rate of the PAF recurrence within the first 3 months after PVI was quite different between the patients with S-PVI and those with C-PVI.

\section{Discussion}

Previous studies have suggested that adding an ablation to the PV ablation aimed at vagal denervation adequate for eliminating vagal reflexes ${ }^{9}$ or targeting the ganglionated plexi in the $\mathrm{LA}^{10}$ may reduce $\mathrm{AF}$ recurrence by modifying the autonomic nervous function. However, it has been demonstrated that the cause of AF recurrence after PVI is firstly because of PV conduction recovery, ${ }^{16-18}$ followed by non-PV AF foci, which are mainly located at the PV antrum. ${ }^{14,19,20}$ The major sites of the occurrence of vagal reflexes or ganglionated plexi in the LA are known to be located at the PV ostium and antrum.., 10 Therefore, in the previous studies, $, 9,10$ the possibility cannot be excluded that additional ablation may have unexpectedly prevented PV conduction recovery and eliminated any non-PV AF foci and vagal denervation might have been a side effect.

This study demonstrated that (1) vagal modification was equally created during the standard segmental and circum- ferential PVI procedures, of which the endpoint was not the loss of vagal reflexes but PV electrical disconnection; (2) the PVI technique and vagal denervation were independent predictors of late PAF recurrence during the entire followup; and (3) vagal denervation also suppressed the PAF recurrence that occurred more than 3 months after PVI. These findings suggest that parasympathetic attenuation after PVI might play a role in preventing late PAF recurrence. The reason why vagal modification after PVI prevented late PAF recurrence remains unclear. As previous studies demonstrated, ${ }^{10-12}$ vagal denervation occurring after PVI was not a permanent, but rather a transient phenomenon in the present study and the changes in the HRV parameters returned to baseline within 6 months. On the other hand, the occurrence of PAF greatly depends on variations in autonomic tone, according to studies using HRV. ${ }^{21}$ A shift toward vagal predominance was essentially observed in patients with PAF triggered by $\mathrm{PV}$ foci. ${ }^{22,23} \mathrm{AF}$ is easily initiated and maintained by parasympathetic stimulation, even in structurally normal hearts, and in mongrel dogs transvascular atrial parasympathetic nervous system modification by RF catheter ablation abolished vagally mediated AF. ${ }^{24}$ Parasympathetic stimulation dramatically shortens the atrial effective refractory period and decreases the wavelength of the atrial reentrant circuits that play an important role in the initiation and perpetuation of AF. ${ }^{25-27}$ In this study, greater vagal denervation resulted in greater suppression of PACs after PVI in patients without any PAF recurrence, as compared with those with PAF recurrence. Therefore, we think that the possible mechanism underlying long-term prevention of PAF recurrence by short-term vagal denervation after PVI might be atrial reverse remodeling which vagal modification enabled by suppressing the PAF triggers early after PVI. It is still controversial which is preferable for preventing late AF recurrence, S-PVI or C-PVI. This study suggested that C-PVI was superior to $\mathrm{S}-\mathrm{PVI}$ in preventing late $\mathrm{PAF}$ recurrence, but that result appeared to depend on the incidence of PV conduction recovery and not on the extent of vagal denervation. These findings suggested that vagal denervation might have played a supplemental role in preventing late PAF recurrence.

In a recent report, the deceleration capacity and acceleration capacity, which are believed to mainly reflect the vagal and sympathetic aspects of autonomic function, respectively, were assessed using a novel signal-processing technology in Holter recordings. ${ }^{11}$ That report demonstrated that the standard S-PVI and C-PVI led to significant impairment of both the deceleration and acceleration capacity. However, in that report, there were no significant differences in the rMSSD or HF, which are standard parameters

Table 7. Results of the Multivariate Analysis

\begin{tabular}{lcccl}
\hline Covariates & Coefficient & P value & OR & 95\%CI \\
\hline Age & 0.032 & 0.235 & 1.032 & $0.980-1.087$ \\
Sex $(0 / 1=$ F/M) & -0.566 & 0.397 & 0.568 & $0.154-2.1001$ \\
AF duration & -0.073 & 0.381 & 0.930 & $0.790-1.094$ \\
Ineffective AADs & 0.142 & 0.492 & 1.153 & $0.769-1.728$ \\
SHD $(0 / 1=$ no/yes) & -1.668 & 0.255 & 0.189 & $0.011-3.332$ \\
LAD & -0.099 & 0.113 & 0.905 & $0.800-1.024$ \\
LVEF & -0.044 & 0.244 & 0.957 & $0.889-1.030$ \\
PVI technique (0/1 =C-PVI/S-PVI) & 1.191 & 0.042 & 3.290 & $1.042-10.394$ \\
ln HF & 0.758 & 0.019 & 2.133 & $1.130-4.027$ \\
LF/HF & -1.920 & 0.034 & 0.147 & $0.025-0.865$ \\
\hline
\end{tabular}

OR, odds ratio; CI, confidence interval. Other abbreviations as in Table 2. 
for assessing parasympathetic nervous activity, before and after PVI. No association between the extent of autonomic impairment and success, in terms of freedom from atrial tachyarrhythmias, could be found. The discrepancy between the new and standard parameters might explain why that study could not elicit such an association.

\section{Study Limitations}

In the recent reports, detailed follow-up using transtelephonic and long-term Holter monitoring has revealed that freedom from AF after an ablation may be overestimated because asymptomatic AF recurrence is overlooked. ${ }^{28,29} \mathrm{In}$ the present study, the cure rate of AF might have been slightly overestimated because intermittent Holter recordings alone were performed as clinical follow-up.

\section{Conclusions}

Vagal denervation can prevent late PAF recurrence independent of the PVI technique.

\section{Disclosure}

There was no financial support for this study.

\section{References}

1. Haïssaguerre M, Shah DC, Jaïs P, Hocini M, Yamane T, Deisenhofer I, et al. Electrophysiological breakthroughs from the left atrium to the pulmonary veins. Circulation 2000; 102: 2463-2465.

2. Pappone C, Rosanio S, Oreto G, Tocchi M, Gugliotta F, Vicedomini $\mathrm{G}$, et al. Circumferential radiofrequency ablation of pulmonary vein ostia: A new anatomic approach for curing atrial fibrillation. Circulation 2000; 102: 2619-2628.

3. Verma A, Marrouche NF, Natale A. Pulmonary vein antrum isolation: Intracardiac echocardiography-guided technique. J Cardiovasc Electrophysiol 2004; 15: 1335-1340.

4. Yamada T, Murakami Y, Muto M, Okada T, Okamoto M, Shimizu T, et al. Computerized three-dimensional potential mapping with a multielectrode basket catheter can be useful for pulmonary vein electrical disconnection. J Interv Card Electrophysiol 2005; 12: 23-33.

5. Arentz T, Von Rosenthal J, Weber R, Burkle G, Blum T, Stockinger $\mathrm{J}$, et al. Effects of circumferential ostial radiofrequency lesions on pulmonary vein activation recorded with a multipolar basket catheter. J Cardiovasc Electrophysiol 2005; 16: 302-308.

6. Yamada T, Murakami Y, Okada T, Okamoto M, Shimizu T, Toyama $\mathrm{J}$, et al. Electrophysiological pulmonary vein antrum isolation with a multielectrode basket catheter is feasible and effective for curing paroxysmal atrial fibrillation: Efficacy of minimally extensive pulmonary vein isolation. Heart Rhythm 2006; 3: 377-384.

7. Yamada T, Murakami Y, Okada T, Yoshida N, Toyama J, Yoshida Y, et al. Pulmonary vein antrum not always coaxial to the pulmonary vein: A dimensional pitfall to the circumferential isolation technique. Circ J 2007; 71: 1430-1436.

8. Miyazaki S, Kuwahara T, Takahashi A, Kobori A, Takahashi Y, Nozato T, et al. Effect of left atrial ablation on the quality of life in patients with atrial fibrillation. Circ J 2008; 72: 582-587.

9. Pappone C, Santinelli V, Manguso F, Vicedomini G, Gugliotta F, Augello G, et al. Pulmonary vein denervation enhances long-term benefit after circumferential ablation for paroxysmal atrial fibrillation. Circulation 2004; 109: 327-334.

10. Scherlag BJ, Nakagawa H, Jackman WM, Yamanashi WS, Patterson E, Po S, et al. Electrical stimulation to identify neural elements on the heart: Their role in atrial fibrillation. J Interv Card Electrophysiol 2005; 13(Suppl 1): 37-42.

11. Bauer A, Deisenhofer I, Schneider R, Zrenner B, Barthel P, Karch M, et al. Effects of circumferential or segmental pulmonary vein ablation for paroxysmal atrial fibrillation on cardiac autonomic function. Heart Rhythm 2006; 3: 1428-1435.

12. Yoshida N, Yamada T, Murakami Y, Okada T, Ninomiya Y, Toyama $\mathrm{J}$, et al. Vagal modification can also help prevent late recurrence of atrial fibrillation after segmental pulmonary vein isolation. Circ $J$ 2009; 73: 632-638.

13. Muto M, Yamada T, Murakami Y, Okada T, Okamoto M, Shimizu T, et al. Electrophysiologic characteristics and outcome of segmental ostial superior vena cava isolation in patients with paroxysmal atrial fibrillation initiated by superior vena cava ectopy: Comparison with pulmonary vein isolation. J Electrocardiol 2007; 40: 319-325.

14. Yamada T, Murakami Y, Okada T, Yoshida N, Ninomiya Y, Toyama $\mathrm{J}$, et al. Non-pulmonary vein epicardial foci of atrial fibrillation identified in the left atrium after pulmonary vein isolation. Pacing Clin Electrophysiol 2007; 30: $1323-1330$.

15. Task Force of the European Society of Cardiology and the North American Society of Pacing and Electrophysiology. Heart rate variability: Standards of measurements, physiological interpretation, and clinical use. Circulation 1996; 93: 1043-1065.

16. Nanthakumar K, Plumb VJ, Epstein AE, Veenhuyzen GD, Link D, Kay GN. Resumption of electrical conduction in previously isolated pulmonary veins: Rationale for a different strategy? Circulation 2004; 109: $1226-1229$.

17. Cappato R, Negroni S, Pecora D, Bentivegna S, Lupo PP, Carolei A, et al. Prospective assessment of late conduction recurrence across radiofrequency lesions producing electrical disconnection at the pulmonary vein ostium in patients with atrial fibrillation. Circulation 2003; 108: 1599-1604.

18. Yamada T, Murakami Y, Okada T, Okamoto M, Shimizu T, Toyama $\mathrm{J}$, et al. Incidence, location, and cause of recovery of electrical connections between the pulmonary veins and the left atrium after pulmonary vein isolation. Europace 2006; 8: $182-188$

19. Lee SH, Tai CT, Hsieh MH, Tsao HM, Lin YJ, Chang SL, et al. Predictors of non-pulmonary vein ectopic beats initiating paroxysmal atrial fibrillation: Implication for catheter ablation. J Am Coll Cardiol 2005; 46: 1054-1059.

20. Scharf C, Oral H, Chugh A, Hall B, Good E, Cheung P, et al. Acute effects of left atrial radiofrequency ablation on atrial fibrillation. J Cardiovasc Electrophysiol 2004; 15: 515-521.

21. Bettoni M, Zimmermann M. Autonomic tone variations before the onset of paroxysmal atrial fibrillation. Circulation 2002; 105: $2753-$ 2759.

22. Zimmermann M, Kalusche D. Fluctuation in autonomic tone is a major determinant of sustained atrial arrhythmias in patients with focal ectopy originating from the pulmonary veins. J Cardiovasc Electrophysiol 2001; 12: 285-291.

23. Liu P, Guo JH, Zhang HC, Wang MX, Li XB, Zhang P, et al. Vagal effects on the occurrence of focal atrial fibrillation originating from the pulmonary veins. Circ J 2009; 73: 48-54.

24. Schauerte P, Scherlag BJ, Pitha J, Scherlag MA, Reynolds D, Lazzara $\mathrm{R}$, et al. Catheter ablation of cardiac autonomic nerves for prevention of vagal atrial fibrillation. Circulation 2000; 102: 2774-2780.

25. Smeets JL, Allessie MA, Lammers WJ, Bonke FI, Hollen J. The wavelength of the cardiac impulse and reentrant arrhythmias in isolated rabbit atrium: The role of heart rate, autonomic transmitters, temperature, and potassium. Circ Res 1986; 58: 96-108.

26. Zipes DP, Mihalick MJ, Robbins GT. Effects of selective vagal and stellate ganglion stimulation on atrial refractoriness. Cardiovasc Res 1974; 8: 647-655.

27. Liu L, Nattel S. Differing sympathetic and vagal effects on atrial fibrillation in dogs: Role of refractoriness heterogeneity. Am J Physiol 1997; 273: $805-816$.

28. Hindricks G, Piorkowski C, Tanner H, Kobza R, Gerds-Li JH, Carbucicchio $\mathrm{C}$, et al. Perception of atrial fibrillation before and after radiofrequency catheter ablation: Relevance of asymptomatic arrhythmia recurrence. Circulation 2005; 112: 307-313.

29. Piorkowski C, Kottkamp H, Tanner H, Kobza R, Nielsen JC, Arya A, et al. Value of different follow-up strategies to assess the efficacy of circumferential pulmonary vein ablation for the curative treatment of atrial fibrillation. J Cardiovasc Electrophysiol 2005; 16: 1286-1292. 